\title{
Zwei neue Technologien für RestoDontics
}

\section{Bei einer endodontischen Behandlung kommt es von der Anam- nese bis zur koronalen Restauration auf jeden einzelnen Schritt an, denn das schwächste Glied bestimmt hier wohl so stark wie in kaum einer anderen Teildisziplin der Zahnheilkunde den Ge- samterfolg. Umgekehrt birgt jede medizinische und/oder tech- nische Innovation entlang der „endodontischen Prozesskette“ die Gelegenheit, sie insgesamt sicherer zu machen. In diesem Sinne werden im Folgenden 2 solche Neuheiten vorgestellt.}

„Endodontie“ ist stets gleich „endodontische Behandlung von A bis Z“ - weit über die Aufbereitung, Reinigung und Füllung von Wurzelkanälen hinaus. So ist heute der Zusammenhang zwischen der eigentlichen endodontischen Behandlung und der koronalen Restauration evident und lässt sich sogar mit harten Zahlen belegen.

\section{Von der Wurzel bis zur Krone $\checkmark$}

Die Erfolgsquote von Zähnen mit guter Wurzelkanalobturation ging bei schlechter koronaler Restauration von circa $90 \%$ auf $44 \%$ zurück [Ray HA, Trope M. Int Endod J 1995; 28: 12-18]. Sieht man sich die Resultate genau an, so muss man zu dem Schluss kommen, dass einem koronal dichten adhäsiven Verschluss eine ähnlich wichtige Rolle wie der Wurzelkanalbehandlung selbst zukommt [Ehrensberger C. ZWR 2014; 123: 237-238].

Darum sind über die letzten Jahre beide Teildisziplinen immer enger zusammengewachsen, teilweise sogar zu zertifizierten Therapiesystemen. So können von der Herstellung des Gleitpfads über die Wurzelkanalaufbereitung bis hin zum PostEndo-Verschluss und der anschließenden direkten oder indirekten Versorgung viele aufeinander abgestimmte Hilfsmittel zum Einsatz kommen ( $\odot$ Abb. 1-4).Zum Instrumentarium zählen heute auch maschinengetriebene Feilen zur Erzeugung des Gleitpfads, kontinuierlich rotierende oder reziprok arbeitende Feilen zur Wurzelkanalaufbereitung und ebenso Adhäsive,
Teilmatrizensysteme, Bulkfüll-Komposite und Deckkomposite - ein weites Feld für Zahnärzte wie für innovative Ingenieure.

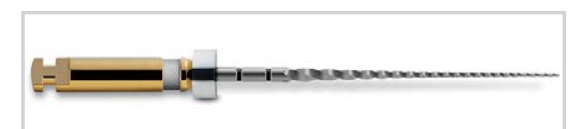

Abb. 1 Hohe Flexibilität, hohe Bruchsicherheit: maschinengetriebene Feile zur Herstellung des Gleitpfads (PRO GLIDER, DENTSPLY Maillefer) (Foto: DENTSPLY Maillefer).

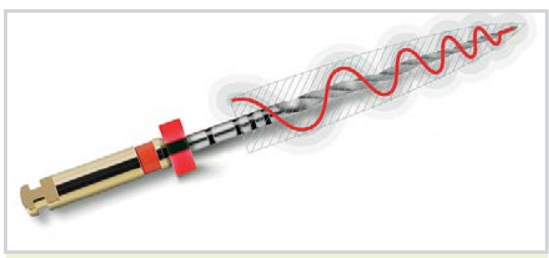

Abb. 2 Bei der Aufbereitung des Wurzelkanals sind maschinengetriebene Feilen schon weit verbreitet - hier ein Beispiel für ein modernes Instrument mit exzentrisch rotierendem Querschnitt (PROTAPER NEXT, DENTSPLY Maillefer) (Foto: DENTSPLY Maillefer).

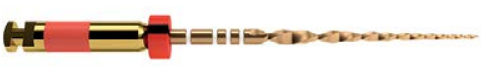

Abb. 3 Reziprok arbeitende Feilen lassen sich besonders einfach handhaben und ermöglichen eine klinisch sichere Aufbereitung binnen kürzester Zeit. Noch mehr Sicherheit, verbesserte Schneidleistung, höhere Flexibilität bietet die neue WAVEONE GOLD. Sie macht in den meisten Fällen die Aufbereitung mit nur einer einzigen Feile möglich (Foto: DENTSPLY Maillefer).

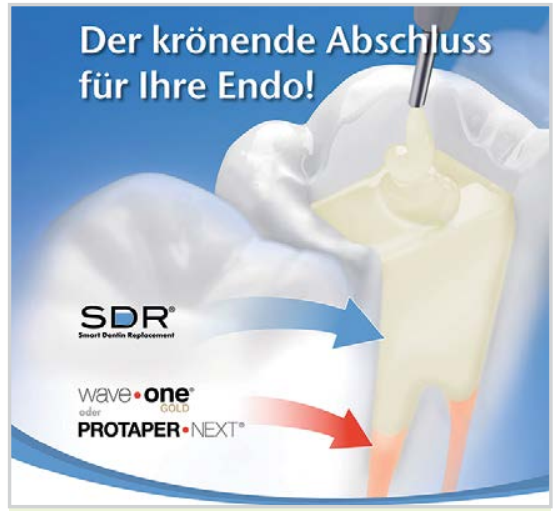

Abb. 4 Ob der Zahnarzt eine kontinuierlich oder reziprok arbeitende Feile verwendet hat - in jedem Falle eignet sich zum sicheren Kavitätenverschluss ein Bulkfüll-Komposit (Foto: DENTSPLY).

\section{Das ist einfach enorm praktisch und vielseitig \\ $\nabla$}

Eine ganz besondere Neuheit und gleichzeitig ein Brückenschlag von der Endodontie in die digitale Welt besteht in einem innovativen Antriebssystem, das sich per iPad steuern lässt und über seine Funktionen als Endomotor hinaus das Praxisteam in allen Behandlungsphasen unterstützt ( $\odot$ Abb. 5; X-SMART iQ DENTSPLY Maillefer).

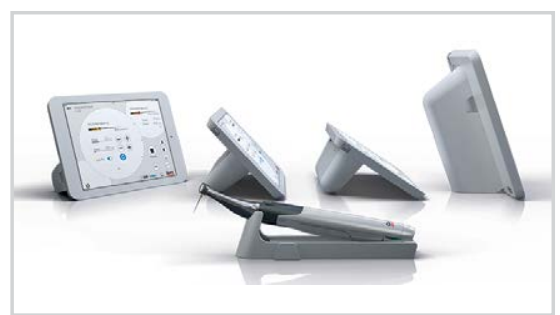

Abb. 5 Einbettung der Endo-Behandlung in die digitale Welt: Der neue Motor X-SMART iQ wird über iPad mit der Apple iOS iQ-App von DENTSPLY Maillefer gesteuert (Foto: DENTSPLY Maillefer).

Es handelt sich dabei um ein kabelloses System, das sowohl für kontinuierliche als auch für reziproke Bewegungen ausgelegt ist. Die Steuerung via iPad erfolgt über die Apple iOS iQ-App von DENTSPLY Maillefer. Diese digitale Plattform bietet vielfältige neue Möglichkeiten von der interaktiven Patientenaufklärung über die Anlage eines individuellen Patientenprotokolls bis zum „Real Time Torque Monitoring” (RTTM). Damit lässt sich das auf die jeweilige Feile wirkende Drehmoment in Echtzeit und über längere Zeiträume visualisieren. 


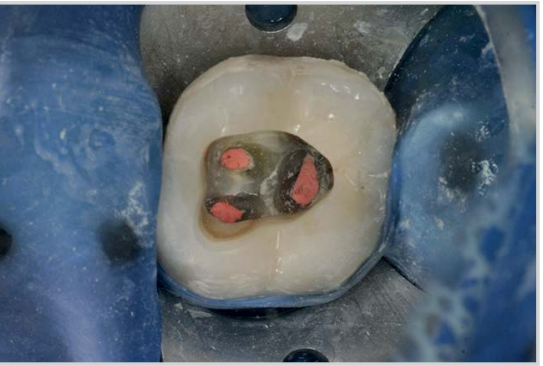

Abb. 6 Zahn 46 unmittelbar nach Obturation mit Guttapercha (Foto: Holzmeier)

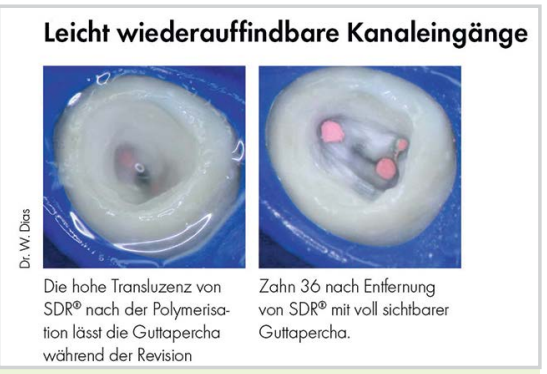

Abb. 8 Wiederauffindbare Kanaleingänge.

Es versteht sich von selbst, dass bei einem solchen System auch die Hygiene und Infektionskontrolle als integraler Hauptbestandteil der zahnärztlichen Berufsausübung stimmen muss. Einen wichtigen Pluspunkt macht der X-SMART iQ in diesem Bereich mit einer maßgeschneiderten Spezialhülle für das iPad. Dieses „Case“ bedeutet ein hygienisches Plus - übrigens auch im Vergleich zu ähnlichen Systemen.

\section{Endo-Verschluss in der Bulkfüll- Technik \\ $\nabla$}

Von der digitalen in die klassisch analoge Welt der Füllungswerkstoffe: Der sichere Post-Endo-Verschluss gelingt heute bei immer mehr Behandlungen mit einem multiindikativen Bulkfüll-Komposit (z.B. SDR, DENTSPLY; $\bullet$ Abb. 6 und 7). Bei diesem Schritt helfen unter anderem spezielle Teilmatrizensysteme für die sichere Herstellung anatomisch korrekter Kontaktpunkte (z.B. Palodent V3, DENTSPLY). Und im Falle einer Revision kann das Bulkfüll-Material die Sicht erleichtern ( $\bullet$ Abb. 8 und 9). Als Deckkomposit zur Gestaltung der Okklusalfläche empfiehlt sich z.B. das nanokeramische Komposit Ceram.X (DENTSPLY; ๑ Abb. 10).

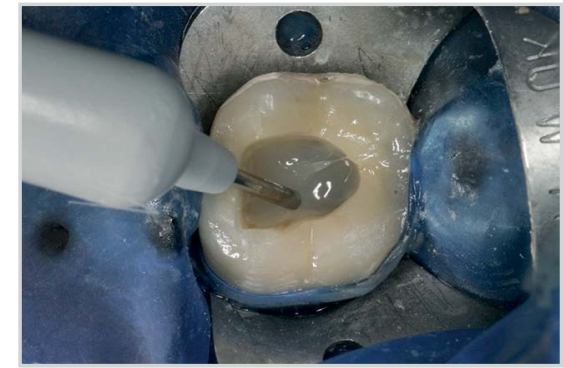

Abb. 7 Bulkfüllung mit Handling-Vorteilen: exzellente Selbstnivellierung und Kavitäten-Adaptation (SDR, DENTSPLY) (Foto: Holzmeier)

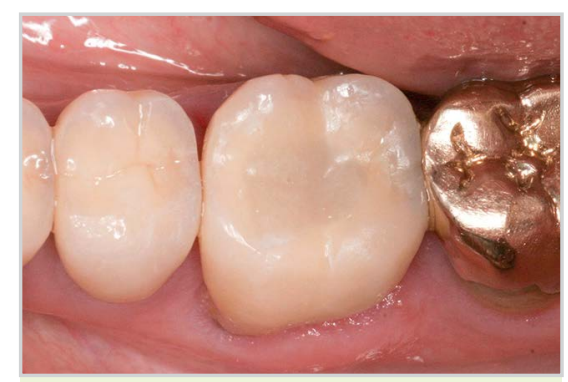

Abb. 9 Finale Situation mit Deckkomposit (Ceram·X mono+, DENTSPLY) (Foto: Holzmeier)

\section{Ein neues universelles nanokera- misches Füllungsmaterial $\nabla$}

Am 1. September kam nun ein neues ceram.x als „universelles nanokeramisches Füllungsmaterial“ auf den Markt. Seine physikalischen und mechanischen Eigenschaften liegen im Vergleich zur bisherigen Version auf einem nochmals deutlich höheren Niveau. Nach dem einfachen Ausbringen aus der Compule weist das neue ceram.x universal eine feste Konsistenz auf und dazu eine hohe Standfestigkeit für ein präzises Modellieren und Ausformen. Dabei lässt es sich sehr gut an die Kavitätenwände adaptieren ohne Verkleben am Modellierinstrument, und auch die abschließende Hochglanzpolitur geht einfach und schnell von der Hand.

Die Ursache für all diese Vorteile liegt in der neuartigen zum Patent angemeldeten „SphereTEC-Füllertechnologie“: Hierfür kommen vorpolymerisierte Füllkörper in Kugelform zum Einsatz. Deren Oberfläche ist allerdings nicht völlig glatt, sondern sie besitzt eine definierte Mikrostruktur.

Es ist alles andere als trivial, solche chemisch und geometrisch komplexen Körper mit der nötigen Kontrollsicherheit herzustellen. Darum kommt hier als Verfahren die Sprühgranulation zum Einsatz: - 1. Schritt: Man versprüht eine Suspension aus Bariumglas, aktiviertem Harz und Lösungsmittel in sehr feine Tröpfchen.

2 2. Schritt: Die Oberflächenspannung zwingt die Tröpfchen in Kugelformen mit wohldefinierter Partikelgrößenverteilung und das Lösungsmittel wird verdampft.

- 3. Schritt: Die vorgeformten Partikel werden polymerisiert. Als Ergebnis erhält man die gewünschten vorpolymerisierten kugelförmigen „SphereTECFüllkörper" mit einem mittleren Durchmesser von etwa $15 \mu \mathrm{m}$.

Einen weiteren Bestandteil der Komposition des neuen Werkstoffs stellt das für die Herstellung der kugelförmigen Granulate verwendete Bariumglas mit einer mittleren Füllergröße von 0,6 $\mu \mathrm{m}$ als nicht agglomerierter Füller dar. Durch diese bimodale Füllergrößenverteilung, bei der die kleineren Glaspartikel die Lücken zwischen den größeren kugelförmigen Granulaten füllen, wird der Füllstoffgehalt optimiert.

Ein weiterer Vorteil der SphereTEC-Partikel ist ihre mikrostrukturierte Oberfläche, durch die mehr freies Harz als durch konventionelle Füllstoffe gebunden werden kann. Dies resultiert in einer geringen Klebrigkeit am zahnärztlichen Instrument.

Hinzu tritt ein sogenannter Kugellagereffekt: Die großen kugelförmigen SphereTEC-Füller in Verbindung mit den unregelmäßig geformten Glaspartikeln führen zu thixotropen Eigenschaften, die für das spürbar leichte Ausbringen aus der Compule, für die gute Adaption an die Kavitätenwände und für die gute Formbarkeit bei hoher Standfestigkeit sorgen.

\section{Ästhetische Restauration - bei Be- darf auch indirekt $\nabla$}

Dank gleichmäßigem Abtrag des ausgehärteten Materials bei der Politur lässt sich schnell und einfach ein hoher Oberflächenglanz erzeugen. Bei der ästhetischen Gestaltung profitiert der Zahnarzt zudem vom neuen CLOUD-Konzept: Dies bringt eine deutliche Vereinfachung mit 


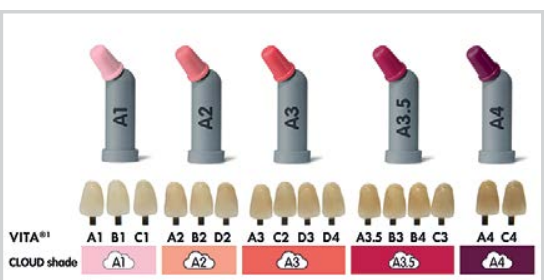

Abb. 11 Die Zuordnung im CLOUD-Konzept: 5 Farben decken das gesamte VITA Classic-Spektrum (eingetragene Marke der VITA Zahnfabrik, Bad Säckingen) ab (Foto: DENTSPLY).

sich. Nur 5 universelle Farben von A1 bis A4 decken das gesamte VITA Classic-Spektrum ab ( $\bullet$ Abb. 11). Ein ausgeprägter Chamäleon-Effekt sorgt dafür, dass sich die Restauration an die Farbgebung des gefüllten Zahns anpasst.

Eine weitreichende Indikationserweiterung besteht in der Ausdehnung des Spektrums von direkten (bestehendes Ceram.X) auf direkte und indirekte (neues ceram.x universal) Füllungen. Die Grundlage dafür liegt in einer hohen Biegefestigkeit, Ermüdungsresistenz, Bruchzähigkeit und Abrasionsfestigkeit dank der SphereTEC-Technologie.

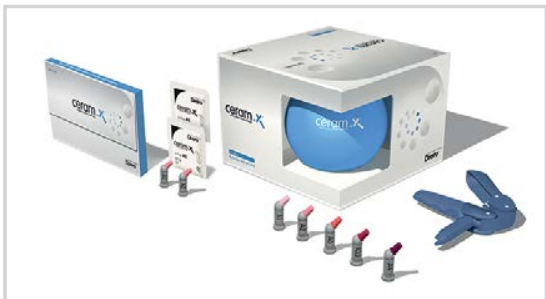

Abb. 12 Zur Markteinführung gibt es die Starterpackung mit 24 Compules in allen 5 Farben zusammen mit einem „Discovery-Kit“ zum risikofreien Ausprobieren: Sollte die neue Ceram·X-Generation wider Erwarten nicht gefallen, kann die Einführungspackung zurückgegeben werden, und der Kaufpreis wird erstattet (Foto: DENTSPLY).

Selbstverständlich kann statt der Kombination „Unterfüllungskomposit SDR + ästhetisches Deckkomposit ceram.x universal“ auch die gesamte postendodontische Restauration mit ceram.x universal erfolgen. Gerade bei indirekten Versorgungen stellt dies eine ganz neue Möglichkeit dar, ohne dass der Behandler auf ein anderes (Spezial-)Material umsteigen müsste.

So bekommt der behandelnde Zahnarzt über den gesamten Therapieverlauf ein

\section{Fazit}

Die endodontische Behandlung schließt eine Reihe unterschiedlicher Schritte ein, und doch müssen sie im Sinne eines maximalen Erfolgs eine Einheit ergeben. Dem wird heute zuweilen mit dem Begriff RestoDontics Ausdruck verliehen. Darunter subsummiert man Techniken der Wurzelkanalbehandlung ebenso wie der restaurativen Zahnheilkunde. Nach Möglichkeit treffen bewährte, aufeinander abgestimmte Systeme zusammen, die aus 1 Hand kommen.

Maximum an klinischer Sicherheit und profitiert gegebenenfalls zusätzlich von modernen ästhetischen Konzepten und Vorteilen beim Handling. In allen Bereichen lassen sich durch gezielte Innovationen Verbesserungen erzielen, wie der vorstehende Blick auf 2 aktuelle Beispiele verdeutlicht - ob sie sich nun im digitalen (X-SMART iQ) oder im klassischen werkstofflichen Bereich (neue ceram.XGeneration, $\triangle$ Abb. 12) bewegen.

Dr. Christian Ehrensberger, Frankfurt/M. 